\title{
Optimization strategies for Microgrid energy management systems by Genetic Algorithms
}

\author{
Stefano Leonori*, Maurizio Paschero, Fabio Massimo Frattale Mascioli, Antonello Rizzi \\ Department of Information Engineering, Electronics and Telecommunications - University of Rome "La Sapienza" - Via Eudossiana \\ 18, 00184, Rome, Italy
}

\section{A R T I C L E I N F O}

\section{Article history:}

Received 16 August 2018

Received in revised form 15 September 2019

Accepted 29 October 2019

Available online 7 November 2019

\section{Keywords:}

Microgrids

Genetic algorithms

Fuzzy systems

Energy management systems

\begin{abstract}
A B S T R A C T
Grid-connected Microgrids (MGs) have a key role for bottom-up modernization of the electric distribution network forward next generation Smart Grids, allowing the application of Demand Response (DR) services, as well as the active participation of prosumers into the energy market. To this aim, MGs must be equipped with suitable Energy Management Systems (EMSs) in charge to efficiently manage in real time internal energy flows and the connection with the grid. Several decision making EMSs are proposed in literature mainly based on soft computing techniques and stochastic models. The adoption of Fuzzy Inference Systems (FISs) has proved to be very successful due to their ease of implementation, low computational run time cost, and the high level of interpretability with respect to more conventional models. In this work we investigate different strategies for the synthesis of a FIS (i.e. rule based) EMS by means of a hierarchical Genetic Algorithm (GA) with the aim to maximize the profit generated by the energy exchange with the grid, assuming a Time Of Use (TOU) energy price policy, and at the same time to reduce the EMS rule base system complexity. Results show that the performances are just $10 \%$ below to the ideal (optimal) reference solution, even when the rule base system is reduced to less than 30 rules.
\end{abstract}

(C) 2019 Elsevier B.V. All rights reserved.

\section{Introduction}

In the last decades, several governments are implementing different energy policies to encompass a sustainable energy future. These policies promote the increasing use of Renewable Energies Sources (RESs), Hybrid Electric Vehicles (HEVs), Plug-in Electric Vehicles (PEVs) and the efficient use of energy [1-3]. However, with the spread of distributed generation from RESs, which is contributing to move the energy generation into the distribution level, the commercialization of PEVs and charging stations, the electric grid will be subject increasing power oscillations and bidirectionalities. These phenomena have to be properly managed in order to keep low the risk of outages and faults, assuring the quality and continuity of the service and avoiding to stress large scale power plants. For example, in [4] a report on the recent trends in PV generation in Germany well highlights the problems caused by the excessive overproduction from PV which forces the temporary closure of coal-fired power plants. In [5] and [6] studies on the impact of Fast and Ultra PEVs charge stations in

\footnotetext{
* Corresponding author.

E-mail addresses: stefano.leonori@uniroma1.it (S. Leonori), maurizio.paschero@uniroma1.it (M. Paschero),

fabiomassimo.frattalemascioli@uniroma1.it (F.M. Frattale Mascioli), antonello.rizzi@uniroma1.it (A. Rizzi).
}

the distribution grid are described, whereas in [7] the authors assert the necessity to strengthen the collaboration between the Distribution System Operator (DSO) and the Transmission System Operator (TSO).

Furthermore to tackle these problems, since the beginning of the 2000s, the United States government has well emphasized the importance of applying Demand Response (DR) programs in order to improve the flexibility of the distribution grid, namely, through the release of special energy price tariffs and incentive programs [2] able to influence and change customers behaviours [8]. DR programs should also support customers to play a more active role in energy market by controlling, scheduling and managing their own loads, especially in presence of local generators and Energy Storage Systems (ESSs) [9]. However, their implementation requires the installation of local Information \& Communication Technology (ICT) infrastructures and power systems able to efficiently monitor, control and manage the customer energy systems and devices. In literature, such kind of paradigm is often referred to as Microgrid (MG). The U.S. Department of Energy defines MGs as a group of interconnected loads and distributed energy resources within clearly defined electrical boundaries, which act as a single controllable entity with respect to the grid. A MG can connect and disconnect from the grid to enable it to operate in both grid-connected and islanded modes [10]. Therefore, during disturbances, the MG generation systems and the local 


\begin{tabular}{|c|c|}
\hline \multicolumn{2}{|c|}{ Adopted Notation } \\
\hline$\Gamma_{\text {big }}$ & $\begin{array}{l}\text { Genetic Algorithm big mutation opera- } \\
\text { tor. }\end{array}$ \\
\hline$\gamma_{\text {conv }}$ & $\begin{array}{l}\text { Genetic Algorithm best fitness mini- } \\
\text { mum improvement threshold used for } \\
\text { the stop condition. }\end{array}$ \\
\hline$\Gamma_{\text {cross }}$ & Genetic Algorithm cross-over operator. \\
\hline$\gamma_{\text {damp }}$ & Mutation rate damping factor. \\
\hline$\Gamma_{\text {del }}$ & Genetic Algorithm delete operator. \\
\hline$\gamma_{\text {dist }}$ & $\begin{array}{l}\text { Genetic Algorithm mutation upper and } \\
\text { lower limit. }\end{array}$ \\
\hline$\gamma_{\text {fit }}$ & $\begin{array}{l}\text { Fitness value associated to a generic } \\
\text { Genetic Algorithm individual. }\end{array}$ \\
\hline$\gamma_{\text {freq }}$ & Mutation rate. \\
\hline$\Gamma_{\text {identity }}$ & $\begin{array}{l}\text { Genetic Algorithm identity operator for } \\
\text { the best individual preservation. }\end{array}$ \\
\hline$\Gamma_{\text {rand }}$ & $\begin{array}{l}\text { Genetic Algorithm operator used to } \\
\text { randomly create new individuals. }\end{array}$ \\
\hline$\gamma_{\text {scale }}$ & $\begin{array}{l}\text { Genetic Algorithm scale factor used by } \\
\text { the big mutation operator. }\end{array}$ \\
\hline$\Gamma_{\text {select }}$ & Genetic Algorithm selection operator. \\
\hline$\Gamma_{\text {small }}$ & $\begin{array}{l}\text { Genetic Algorithm small mutation oper- } \\
\text { ator. }\end{array}$ \\
\hline$\Gamma_{\text {stop }}$ & $\begin{array}{l}\text { Genetic Algorithm stop condition oper- } \\
\text { ator. }\end{array}$ \\
\hline$\gamma_{\text {th }}$ & $\begin{array}{l}\text { Genetic Algorithm upper fitness thresh- } \\
\text { old used by the delete operator. }\end{array}$ \\
\hline$\Omega$ & FIS parameters set. \\
\hline$\Theta_{C A}$ & $\begin{array}{l}\text { FIS overall rule consequents association } \\
\text { subset. }\end{array}$ \\
\hline$\Theta_{M F}$ & FIS Membership Functions subset. \\
\hline$\Theta_{M H}$ & FIS rule hierarchy subset. \\
\hline$\Theta_{R W}$ & FIS Rule Weights subset. \\
\hline$b_{i}$ & $\begin{array}{l}\text { Element of the subset } \Theta_{M H} \text { for the } \\
\text { activation of the } i \text { th rule. }\end{array}$ \\
\hline$N_{\text {conv }}$ & $\begin{array}{l}\text { Number of generations of stall for } \\
\text { the Genetic Algorithm stop criteria } \\
\text { activation. }\end{array}$ \\
\hline$N_{\text {del }}$ & Number of deleted individuals. \\
\hline$N_{\text {elite }}$ & $\begin{array}{l}\text { Number of elite individuals for each } \\
\text { Genetic Algorithm generation. }\end{array}$ \\
\hline$N_{\text {ind }}$ & Genetic Algorithm population size. \\
\hline$N_{\text {in }}$ & Number of FIS input. \\
\hline$N_{M F}$ & $\begin{array}{l}\text { Maximum number of MFs composing } \\
\text { the FIS. }\end{array}$ \\
\hline$N_{m f}$ & $\begin{array}{l}\text { Maximum cardinality of each Fuzzy } \\
\text { Term Set. }\end{array}$ \\
\hline$N_{M H}$ & $\Theta_{M H}$ cardinality. \\
\hline$N_{\text {rules }}$ & Maximum number of Fuzzy rules. \\
\hline$N_{\text {stop }}$ & $\begin{array}{l}\text { Genetic Algorithm maximum number } \\
\text { of generations. }\end{array}$ \\
\hline
\end{tabular}

loads can separate from the distribution maintaining high level of service without harming the transmission grid integrity [11].

A MG can be designed to cover a single dwelling (e.g. smart home MG) up to an entire district. Moreover, also HEVs can be considered particular MGs since they are equipped with auxiliary or multi-generation power units capable to keep high dynamic response performances in case of breaking or acceleration. Because of their smaller dimensions they are usually referred to

\begin{tabular}{|c|c|}
\hline$N_{T}$ & $\begin{array}{l}\text { Number of participants to each Genetic } \\
\text { Algorithm tournament selection. }\end{array}$ \\
\hline$P$ & Genetic Algorithm Objective Function. \\
\hline$p_{d e l}$ & $\begin{array}{l}\text { Uniformly distributed probability of be- } \\
\text { ing deleted for those individuals fea- } \\
\text { tured by a fitness above a given thresh- } \\
\text { old } \gamma_{t h} \text {. }\end{array}$ \\
\hline$q_{i}$ & $\begin{array}{l}\text { Element of the subset } \Theta_{R C} \text { for the } i \text { th } \\
\text { rule consequent definition. }\end{array}$ \\
\hline$w_{i}$ & Rule weight associated to the $i$ th rule. \\
\hline
\end{tabular}

as "nano-grids" which mostly operate in islanded mode [12], i.e. disconnected from the charging station [13-15].

About the MG electrical architecture, it is usually composed by a main bus known as backbone which allows the connection of the distributed energy systems power converters (i.e. loads, generators and ESSs) and the main grid. It can be designed both in DC and AC, as well as in ring or radial mode [16]. In [17,18] authors discuss the advantages of installing DC MGs with respect to $\mathrm{AC}$ MGs due to the simpler control and the reduction of the conversion stages, especially in PV systems, batteries and DC loads including the charging of PEVs. Furthermore, the same authors affirm that such advantages will bring to the gradual transformation of the distribution grid in DC. However, more recent studies state that hybrid AC-DC MGs seem to be more promising due to protection, reliability and quality of power reasons, as well as the possibility of exploiting both the benefits given by $A C$ and $D C$ MGs. Indeed, considering residential MGs, hybrid AC-DC architectures would avoid modifications in the electrical installation that supplies energy to the apartments [19, 20].

Concerning MG energy flows management, as stated in [21] where is claimed the MG key-role for the smart grids realization, MG Energy Management Systems (EMSs) belong to the second layer of smart grid control scheme, where the first layer is referred to the control system of each single power device (converters) and the third has to coordinate a cluster of interconnected MGs by interacting with each single MG EMS of the same smart grid. The EMS is thus in charge to implement a real time decision making strategy in order to efficiently control and manage the MG energy flows as well as the connection with the grid. Said in other words, the EMS coordinates the local MG subsystems in order to satisfy the prosumer and DSO needs according to a proper formulated Objective Function (OF). In addition, its operational time frame is dependent on the smart meter sampling time which ranges between few minutes to maximum one hour [8].

Concerning the of formulation, it can be composed of one or more penalty(reward) functions depending on the simulation scenario taken into consideration. These usually are formulated to evaluate the stress on the distribution grid caused by energy flows fluctuations, the MG auto-consumption, peak shaving operations, the profit generated by the energy trading with the grid and the minimization of the operational costs $[8,19,22]$.

About the EMS modelling instead, several approaches have been investigated in the literature ranging from simple rulebased methods which rely on knowledge of expert operators to the adoption of machine learning and data driven techniques. In [23] for instance, three main categories of EMS modelling have been distinguished: heuristic, stochastic and soft computing based models, although many solutions proposed in the literature foresee a hybridization of these approaches. In addition, reinforcement learning approaches have been explored [24-26]. 
The rational behind the investigation of such complex solutions based on data driven techniques in EMS modelling is due to the fact that only in rare cases the EMS can determine an optimal schedule of the local MG energy flows in real time. To this aim, raw implementations of (pseudo)deterministic algorithms based on Linear Programming (LP), Mixed Integer Linear Programming (MILP), greedy algorithms, or Dynamic Programming (DP) are usually reported in literature. In [27] for example, the authors consider a fixed energy price tariff on both the energy bought and sold and the $\mathrm{OF}$ is formulated in order to maximize the incomes generated by the energy trading with the grid. Therefore, the EMS problem has been easily solved through a simple LP formulation.

However, it is important to note that in most cases discussed in literature, the EMS requires the knowledge of the future energy systems time series (e.g. overall energy demand, generation from RESs, time arrival and time departure of PEVs) in order to optimally schedule the MG energy flows, which are characterized by intermittent and stochastic behaviours. Also in automotive applications, EV EMSs show same problem to know the vehicle's route and traffic information. Therefore, as also suggested in [28], the direct application of optimization algorithms in EMS can be tricky and is more suitable for benchmark tests [19,29], EMS reference solution evaluation [14,30] and the dimensioning of energy systems [31].

A very conventional solution well known in literature, which in [32] is even called "classical", consists in the implementation of Rolling Time Horizon (RTH) strategies. It is based on the coupling of a proper formulated optimization algorithm (LP, MILP...) with energy systems prediction algorithms. In particular, RTH based EMSs consist in running at each time sample the prediction algorithms to obtain estimations used by the optimization algorithm in order to compute the optimal output (command) time series over the given time horizon. Once the optimal solution is calculated, only the first element is communicated to the MG power converters in order to effectively balance the MG power flows. Usually, the characteristic time horizon of both optimization and prediction algorithms is featured by one or two days [33,34].

Although RTH strategies have been proven to be very effective and robust in many case studies, they suffer from the dependence on the prediction accuracy and the weak level of interpretability of the decisions taken by the EMS, which makes them black box models. It is worth noting that in case of complex multi-energy systems MGs featured by several controllable energy systems such as shiftable loads, combined heating, power generation units and ESSs, the time interval required by the EMS to define the energy flows can get close to the smart meter sampling time as remarked in [22], where is criticized the use of DP optimization, and shown in [35] where a RTH Markov decision process based EMS is investigated. Furthermore, when the optimization problem is featured by a multi-objective function, the linear combination of the cost functions requires a proper setting of weights (as meta-parameters of the OF) and can be ineffective. In [36] for example, a multi-objective problem is considered in order to minimize both the energy exchanged with the grid and its energy oscillations, while ensuring the fulfillment of given constraints for a correct use of the ESS. In this work, the authors avoided to combine the cost functions since their different nature.

Regarding the choice of a prediction algorithm applied to energy systems time series dedicated to RTH strategy implementation, beside the conventional algorithms as ARIMA, ARMA and ARMAX, most of machine learning models proposed in literature are often based on Neural Networks (NNs) and Fuzzy Systems as remarked in [32], where a critical review on MG EMS methods, solutions and prospects is presented. Moreover in $[37,38]$ authors well highlighted that most of recent research activities are specifically inspired on NNs models. In particular,
Long Short Term Memory (LSTM) NNs [39-42], Echo State Networks (ESNs) [43,44], Fuzzy Inference Systems (FISs) and Adaptive Neuro Fuzzy Inference Systems (ANFIS) $[45,46]$ revealed to be successful solutions.

Besides RTH strategy applications, other approaches are investigated in the literature in order to avoid the high dependency to prediction systems. In [47] and [22] chance constrained scheduling approach have been proposed in order to better deal with the uncertainties of the MG energy systems. Also the adoption of meta-heuristic approaches revealed very effective [32,48,49]. In [14] instead a NN based decision making system has been compared to a simpler rule based EMS. Nevertheless, it is worth noting that even in these cases (i.e. with the adoption of NNs, heuristics and stochastic models) the EMS models still hold a low level of interpretability.

On the contrary, rules-based systems such as FISs can be a viable alternative for their higher level of interpretability and ease of implementation.

Indeed, FISs can be classified as grey-box models, since the adoption of linguistic variables, Fuzzy sets, linguistic operators and "IF-THEN" rules allow to extract and interpret explicit useful knowledge about the decision process, as well to exploit some possible a priori knowledge [50,51].

Moreover, with the capillary diffusion of MGs the EMS computational burden can be another important aspect to consider in EMS modelling. In [52] for instance, authors validated the implementation of a heuristic based EMS co-located on a small smart meter with limited memory and processing power. The complex structure of RTH EMS which relies on the combination in cascades of prediction and optimization algorithms can be replaced by lighter and simpler decision-making systems such as FISs and NNs especially when these show similar or even better performances.

In literature and industrial application, FISs are mainly proposed as decision support systems for control and energy systems prediction as mentioned above. In [53,54] for example FISs are used for the enlightenment and HVAC control in smart homes. In that works the controllers are designed in order to support a more complex EMS which has to shift and schedule the smart home appliances in order to reduce the peak to average ratio and preserve the user comfort. In [55] a type-2 FIS trained by extreme learning machine techniques, known as Extreme Learning-Adaptive Neuro Fuzzy Inference System (EL-ANFIS) [56], is proposed for energy demand forecasting. The solution proposed has been compared with more conventional models, in particular a Feed Forward NN of 10 hidden layers and an ANFIS.

Knowledge based FISs are implemented in [57-59] as MG EMSs for minimizing the economic costs, in [59-61] for reducing abrupt changes in energy flows with the grid and in HEVs for the fuel consumption minimization [15], respectively. However, FISs defined by expert operators knowledge may suffer from loss of accuracy for ill-known or data-intensive models that is the main incentive for using Fuzzy rules inferred from data. As stated in [50], such loss of accuracy can be reduced by nature inspired methods such as Evolutionary Algorithms (EAs) which would preserve the FIS interpretability. For example, in [62] and [63] the effectiveness in tuning the EMS FISs Membership Function (MFs) and Rule Consequences through EAs was analysed. In [30,43,64], a FIS EMSs has been modelled by means of a $k$-means based clustering algorithm. The use of clustering algorithms allowed to efficiently define a small number of multi-variate Gaussian MFs. In [43] is investigated the effectiveness of considering a raw estimation of the future prosumer energy balance trends supporting the FIS decision making system.

Following previous works $[62,65]$, in this paper it is proposed a FIS based EMS able to manage in real time the energy flows 


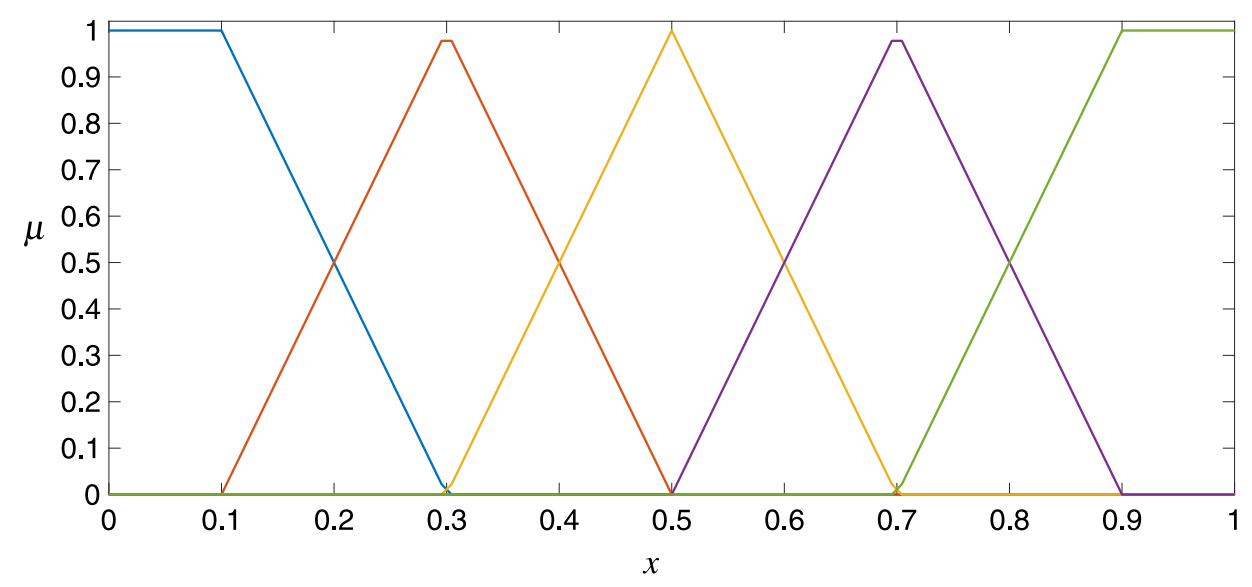

Fig. 1. Term Set initial structure for $N_{m f}=5$.

of a grid connected MG equipped with an ESS. In particular, it is in charge to define how to split the prosumer energy balance between a local ESS and the connected grid in order to maximize the profit generated by the energy trading with the main grid, assuming a Time Of Use (TOU) energy price policy. The EMS is synthesized through a hierarchical Genetic Algorithm (GA)-FIS paradigm which is in charge to define the FIS consequent part of each rule, tune the Membership Functions (MFs) position and shape, set the rule weights and delete those input MFs considered ineffective. Once designed the EMS and set its optimization parameters, different strategies in tuning the FIS parameters are implemented. Results obtained are compared with the optimal reference solution found through a LP algorithm considering known a priori the overall prosumer energy profiles and a benchmark solution based on a RTH strategy.

The paper is organized as follows. In Section 2 it is introduced and explained the hierarchical GA-FIS paradigm, while Section 3 deals with the proposed grid connected MG model and the related problem formulation. Section 3.2 describes in details the EMS synthesis procedure. In Section 4 are illustrated the simulation settings, followed by the results discussed in Section 5. In this section are also described the optimal reference solution (see Section 5.1) and the RTH benchmark solution (see Section 5.2). Finally conclusions are reported in Section 5.

\section{GA-FIS hierarchical optimization paradigm}

The adoption of heuristics for the synthesis and optimization of inference systems through data driven approaches is a topic widely explored in literature. In particular, GA-FIS optimization paradigm has proved to be a very effective tool because of its flexibility and low implementation cost on embedded systems. In GA-FIS paradigm, a specific set of FIS parameters are properly tuned by a GA according to one or more given OFs. In case of multiple OFs the problem is referred as multi-objective GA optimization. In this work, a hierarchical GA optimization is used for the synthesis of a FIS-based MG EMS.

In hierarchical GAs, two class of genes are generally distinguished, namely control genes and parametric genes imitating the functional distinction between regulatory and structural genes, respectively, in DNA. In our case, whereas parametric (structural) genes correspond to the FIS parameters, control genes (regulatory sequences) are in charge to activate or deactivate a given set of parametric genes. In this work in particular, control genes are dedicated to activate or deactivate FIS MFs which are defined by subsets of parametric genes [66]. The hierarchical optimization procedure is designed to both improve the FIS effectiveness and decrease the number of FIS parameters, namely its complexity and computational burden.

In the following of this section, the main FIS structure is described and all the optimization parameters defining the FIS are listed and commented in order to explain their functionality and to better understand how the FIS synthesis process works. At the end of this section, it is introduced and detailed the GA adopted, describing each operator performed during the optimization procedure.

\subsection{FIS structure}

Mamdani Type Fuzzy rules have been adopted for FIS synthesis. Each Term Set is initially composed by $N_{m f}$ symmetric MFs having triangular or trapezoidal (at the domain boundaries) shape characterized by $50 \%$ overlap as illustrated in Fig. 1 for $N_{m f}=5$.

Assuming that the FIS has $N_{\text {in }}$ inputs and a single output, every rule is characterized by $N_{\text {in }}$ antecedents. Each rule antecedent set must consider one (and only one) MF for each Term Set. Therefore, the maximum number of rules $N_{\text {rules }}$ is given by all the possible antecedents combination, namely $N_{\text {rules }}=N_{m f}^{\left(N_{\text {in }}\right)}$.

The structure of the $i$ th Fuzzy rule can be written as

$$
\begin{aligned}
& \text { If }\left(x_{1} \text { is } A_{i 1}\right) \text { and }\left(x_{2} \text { is } A_{i 2}\right) \text { and...and }\left(x_{N_{i n}} \text { is } A_{i N_{i n}}\right) \text { then } \\
& \qquad y \text { is } B_{i}, \quad i=1,2, \ldots, N_{\text {rules }}
\end{aligned}
$$

where, $x_{j}, \mathrm{y}, A_{i j}$ and $B_{i}$ are the $j$ th input, the output, the MF associated with the $j$ th antecedent and the MF associated with the consequent of the $i$ th rule, respectively.

In case of MF deletion or deactivation, the rules including that MF is deleted too, simplifying the rule base. The activation of each rule is regulated by a specific rule weight parameter $w_{i} \in$ $(0,1]$ (for $i=1, \ldots, N_{\text {rules }}$ ). It is employed for the Fuzzy output evaluation by considering the Center Of Area defuzzification method.

In case of a MF deactivation, the MF deleted could leave uncovered intervals in the corresponding input domain. In fact, triangular and trapezoidal MFs, unlike Gaussian or Bell MFs, are characterized by finite supports. For this reason, it has been imposed that, whenever a MF is deactivated, the nearest base points of the adjacent (still active) MFs are crossed and placed under the vertex of the corresponding new adjacent Fuzzy Set, in order to ensure the covering of the overall Fuzzy domain and a given fixed overlap (50\%) of the adjacent MFs. In each Fuzzy domain the number of MFs in the related Term Set must be equal or greater than two, a constraint that has been introduced in the synthesis procedure.

In the following it is described a procedure to optimize the whole set of FIS parameters. 


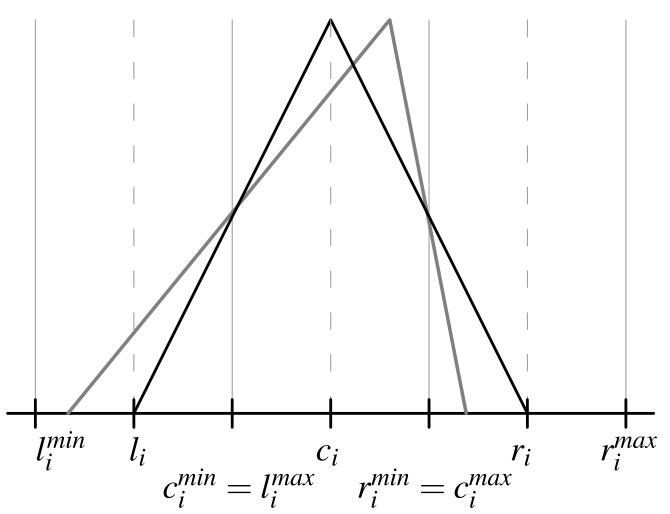

Fig. 2. Parameters and related ranging intervals defining the limits of the triangular MF in black. In grey an example of its possible mutation.

\subsection{FIS parameters}

The set of all the FIS parameters is indicated by $\Omega$. It is composed by four subsets named $\Theta_{M F}, \Theta_{R W}, \Theta_{C A}$ and $\Theta_{M H}$, representing the MFs shape, the rule weights, the rules consequents association and the MF activation, respectively.

The four subsets of parameters are described in details in the following paragraphs.

- Subset $\Theta_{M F}$

The subset of the MFs parameters $\Theta_{M F}$ contains the information about the FIS MFs shape and position. According with [67], each triangular MF is described by three parameters each one ranging in a specific interval of its support domain. More precisely, the ith triangular FIS MF is described by means of $l_{i}, c_{i}$ and $r_{i}$, representing the abscissas that define the base and height of the triangle (see Fig. 2). They range between their related upper and lower bound named min and max, respectively, as illustrated in Fig. 2. The MF upper and lower bound are expressed in terms of $l_{i}, c_{i}$ and $r_{i}$ as stated in (2)

$$
\begin{aligned}
l_{i}^{\text {min }} & =l_{i}-\frac{c_{i}-l_{i}}{2} \\
c_{i}^{\text {min }}=l_{i}^{\text {max }} & =l_{i}+\frac{c_{i}-l_{i}}{2} \\
r_{i}^{\text {min }}=c_{i}^{\text {max }} & =r_{i}-\frac{r_{i}-c_{i}}{2} \\
r_{i}^{\text {max }} & =r_{i}+\frac{r_{i}-c_{i}}{2}
\end{aligned}
$$

This formulation is extended to all the FIS MF including the trapezoidal ones. Being all the MF domain supports defined in $[0,1]$, those MFs of trapezoidal shape at the edges of the support domain can be defined by two points (see Fig. 1). Since each Term Set is composed by 3 triangular and 2 trapezoidal MFs, therefore 13 points, the $\Theta_{M F}$ cardinality is equal to 13 times the overall number of FIS Term Sets (i.e. $\left.\left(N_{\text {in }}+1\right) \cdot 13\right)$. More precisely, the subset of MF parameters is given by $\Theta_{M F}=\left\{l_{1}, c_{1}, r_{1}, l_{2}, c_{2}, r_{2}, \ldots, l_{N_{M F}}, c_{N_{M F}}, r_{N_{M F}}\right\}$, where $N_{M F}$ is the maximum number of MFs composing the FIS $\left(N_{M F}=N_{m f}\left(N_{i n}+1\right)\right)$. The $\Theta_{M F}$ parameters are initially set to generate the FIS Term Sets as shown in Fig. 1.

- Subset $\Theta_{R W}$

The subset of the FIS rule weights parameters $\Theta_{R W}$ includes one real number for each rule, weighting the relevance of each single rule with respect to the others. More precisely, each weight $w_{i}$ ranges in $(0,1]$. The exclusion of the zero value averts the possibility that the FIS takes no decision. This ensures the coverage of the overall input domain by the inference surface, avoiding uncovered subsets of the overall input space.
The cardinality of $\Theta_{R W}$ is equal to the maximum number of rules $N_{\text {rules }}$ allowed for a FIS composed by $N_{\text {in }}$ Term Sets, each one initialized as in Fig. 1. The rule weight values are initially set equal to 1 in order to equally attribute the same importance to each rule. Summarizing, the subset of the FIS rules weighting parameters is given by $\Theta_{R W}=$ $\left\{w_{1}, w_{2} \ldots, w_{N_{\text {rules }}}\right\}$.

- Subset $\Theta_{R C}$

The subset of the rule consequents parameters $\Theta_{R C}$ contains one element for each rule, which specifies the MF selected among the output Term Set. More precisely, the element $q_{i}$ of the subset $\Theta_{R C}$ is an integer number ranging from one to $N_{m f}$ (i.e. the cardinality of the output Term Set). The subset of the FIS rules consequent association parameters is given by $\Theta_{R C}=\left\{q_{1}, q_{2}, \ldots, q_{N_{\text {rules }}}\right\}$. In this work, it is not assumed the presence of an expert operator aware on the problem under analysis, therefore the definition of the initial value of $\Theta_{R C}$ is hard to find. For this reason, the $\Theta_{R C}$ initial state will be randomly generated and therefore optimized, assigning to this step the maximum priority since it constitutes the FIS knowledge core.

- Subset $\Theta_{M H}$

$\Theta_{M H}$ subset includes one binary element for each designed Fuzzy Set in order to establish its activation state. More precisely, the $i$ th binary element $b_{i}$ of the subset $\Theta_{M H}$ is used to switch on or off the corresponding MF in the related input Term Set. The $\Theta_{M H}$ cardinality is given by the total number of possible antecedentes Fuzzy Sets $N_{M H}=N_{i n} N_{m f}$. Summarizing, the subset of the FIS rules hierarchy parameters is given by $\Theta_{M H}=\left\{b_{1}, b_{2}, \ldots, b_{N_{M H}}\right\}$. The $\Theta_{M H}$ initial state foresees the activation of all the Fuzzy Sets.

\subsection{Genetic algorithm}

The genetic code coincides with the FIS parameters set $\Omega=$ $\left\{\Theta_{R C}, \Theta_{M F}, \Theta_{R W}, \Theta_{H R}\right\}$, accordingly in the following the two meanings are attributed to the symbol $\Omega$ equivalently. Due to the heterogeneous nature of the elements belonging to the $i$ th subset $\Theta_{i}$, the generic gene can be a real, an integer or a binary number. All the genes are constrained by their respective upper and lower bound as described previously in Section 2.2. In this work it is assumed that genes corresponding to the parameters belonging to each of the four subsets can be optimized simultaneously or sequentially. In the latter approach, the overall genetic variables set is optimized in subsequent optimization steps. During each step the genes belonging to one or more subsets $\Theta_{i}$ are optimized simultaneously, whereas the remaining ones are kept unchanged, until all genes of the genetic variables set are optimized. It is assumed that each subset can be optimized only once during the entire optimization process. Consequently, once the parameters belonging to a given subset $\Theta_{i}$ are optimized, the corresponding genes are frozen for the rest of the GA optimization process. The FIS subsets not yet optimized are set to their initial state defined in the previous section. It is important to remark that $\Theta_{R C}$ set must be optimized in the first step in case of a sequential optimization. The number of variables simultaneously optimized during each step depends on the cardinality of the specific subset (or subsets) $\Theta_{i}$ considered in the specific optimization step. Once the initial population $G_{0}$ is set, evaluated and sorted according with a given OF $P$, each step of the optimization process is subjected to the following sub-steps repeated for each new generation until the GA stops. Once a new generation is created, individuals belonging to the previous generation are no more taken into consideration. In the following all the details concerning each sub-step of the evolutive optimization process are illustrated. For each sub-step the number of individuals of the $k$ th generation $G_{k}$ is fixed equal to $N_{i n d}$. 


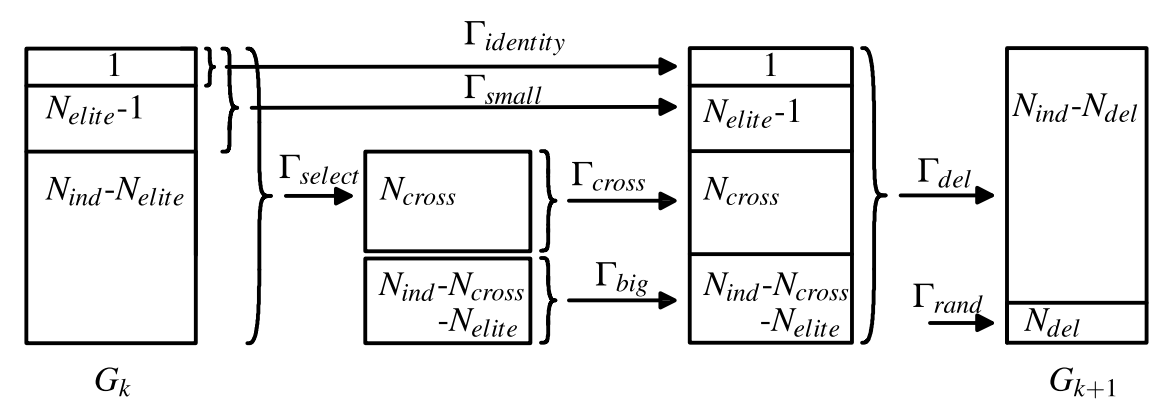

Fig. 3. GA sub-step optimization procedure schematic representation, performing the computation of generation $G_{k+1}$ starting from $G_{k}$.

i The best $N_{\text {elite }}$ individuals are selected, the best one is handed down to the next generation by the $\Gamma_{\text {identity }}$ operator, whereas the remaining $N_{\text {elite }}-1$ are subjected to a small mutation defined by the $\Gamma_{\text {small }}$ operator.

ii Starting from the current generation, an even number $N_{\text {cross }}$ of individuals are chosen by the selection operator $\Gamma_{\text {select }}$ and coupled in $N_{\text {cross }} / 2$ pairs. Each couple is then subjected to the cross-over operator $\Gamma_{\text {cross }}$.

iii Among the unselected individuals of phase-ii the best $N_{\text {ind }}-$ $N_{\text {elite }}-N_{\text {cross }}$ are extracted. Before of being integrated into the new offspring these individuals, which were supposed to be the best among the worst or at least a considerable part of them, are subjected to the big mutation operator $\Gamma_{\text {big }}$.

iv The new offspring is then simulated in order to compute the objective (fitness) function $P$, sorting the population at hand according to $P$.

$\mathrm{V}$ Individuals having a fitness greater than a fixed threshold $\gamma_{\text {th }}$ are subject to the deletion operator $\Gamma_{\text {del }}$ which calculates their deletion probability.

vi The $N_{\text {del }}$ deleted individuals are substituted by new individuals randomly generated by the operator $\Gamma_{\text {rand }}$.

Each sub-step of the GA optimization process is arrested when the convergence is reached. The stop criteria is denoted with the $\Gamma_{\text {stop }}$ operator symbol.

A schematic representation of the sub-steps performed by the GA in order to evaluate a new generation is given in Fig. 3.

In the following a detailed description of the genetic operators is given:

- Operator $\Gamma_{\text {select }}$

The $\Gamma_{\text {select }}$ operator is a tournament selection operator. The $N_{T}$ value defines the number of participants to each tournament.

- Operator $\Gamma_{\text {cross }}$

The $\Gamma_{\text {cross }}$ operator is a scattered cross-over operator. It is applied to a couple of individuals randomly chosen by those that are previously selected. This procedure is repeated until all the selected individuals have been crossed.

- Operator $\Gamma_{\text {identity }}$

The $\Gamma_{\text {identity }}$ operator copies individuals in the next generation without altering their genes.

- Operators $\Gamma_{\text {small }}$ and $\Gamma_{\text {big }}$ When an individual is subject to $\Gamma_{\text {small }}$ or $\Gamma_{\text {big }}$ operator their genes have a distributed probability of being mutated equal to $\gamma_{\text {freq }}$. The mutation consists in altering the selected gene by adding a random real number extracted from a uniform distribution between the interval $\left[\gamma_{\text {dist }},-\gamma_{\text {dist }}\right]$ in case of $\Gamma_{\text {small }}$. For $\Gamma_{\text {big }}$ the interval is extended by an integer scale factor $\gamma_{\text {scale }}$ in order to enhance the exploration. The $\gamma_{\text {dist }}$ value is expressed in percentage with respect to the difference between the respective upper and lower bound of each gene. Integer variables are subjected to the same mutation operators. In addition, they are successively rounded in order to satisfy their constraints. Mutation rates, both big and small, decreases generation by generation thanks to a specific exponential factor $\gamma_{\text {damp }}$ applied to $\Gamma_{\text {small }}$. The mutation distances (i.e. $\gamma_{\text {dist }}$ ) are then exponentially reduced and at the generation $N_{\text {stop }}$ they vanish. This procedure aims at encouraging the exploitation at the expense of the exploration capability with the increasing of the generations.

- Operator $\Gamma_{\text {del }}$

The operator $\Gamma_{\text {del }}$ assigns the probability $p_{\text {del }}$ to delete an individual characterized by a fitness $\gamma_{\text {fit }}$, as follows:

$p_{\text {del }}= \begin{cases}1-e^{-\frac{\left(\mid \gamma_{t h}-\gamma_{\text {fit }}\right) \mid}{\gamma_{\text {th }}}} & \text { if } \gamma_{\text {fit }}>\gamma_{\text {th }} \\ 0 & \text { otherwise. }\end{cases}$

Therefore, the $N_{d e l}$ deleted individuals are replaced by other $N_{d e l}$ new individuals randomly generated. The new individuals are spawn by choosing the value of each gene considering a uniform distributed probability between their respective upper and lower bounds.

- Operator $\Gamma_{\text {stop }}$

It defines the stop conditions of each GA sub-step optimization. If after $N_{\text {conv }}$ consecutive generations it is not observed any improvement on the best fitness greater than a certain percentage $\gamma_{\text {conv }}$ of the maximum number of allowed generations $N_{\text {stop }}$, the GA stops in advance the sub-optimization process. The process stops anyway after $N_{\text {stop }}$ generations.

\section{Application of GA-FIS paradigm to EMS synthesis}

This section is dedicated to the application of the GA-FIS paradigm previously introduced to the synthesis of a FIS based EMS in a MG connected to the electric power distribution infrastructure. First, the MG architecture is described. Then the implementation of the EMS through the GA-FIS is detailed.

\subsection{MG architecture}

In this work it is taken into consideration a grid connected MG equipped with an ESS, loads and local generation. These subcomponents are represented as aggregated systems referred to as: the generation $G$, the load $L$, the ESS $S$ and the main grid $N$. The MG must control and manage the power flows of the aggregated systems, while satisfying given constraints set by the main grid and ensuring the services requested by the prosumer (i.e. the owner of such energy systems, as he acts both as producer and consumer).

The proposed model is based on several hypotheses that define a useful level of abstraction to correctly place the problem under analysis. MG aggregated systems power flows are discretized in time slots of 15 minutes. In each time slot the power level is considered constant. Low level operations such as voltage and reactive power control are not considered. The power 
transmission losses within the MG are considered negligible. The online control module ensure that the power balance is achieved during the real-time operation. The EMS works with a sample time considerably greater than the characteristic time of the ESS power control, therefore the ESS inner loop has been neglected. The power converters which connects the MG sub-components to each other, included that one which allow the MG-grid connection, are neglected in terms of power losses and characteristic time of control.

For the sake of ease, in each time slot the aggregated power flows are reported in terms of energy flows (i.e. exchanged $k W h$ ). The aggregated energy flows are indicated with the terms $E_{k}^{G}$ (generation), $E_{k}^{L}$ (load), $E_{k}^{S}$ (ESS) and $E_{k}^{N}$ (grid) in each $k$ th time slot.

It is assumed that the prosumer energy production $E_{k}^{G}$ has the priority to satisfy in each time slot the prosumer energy demand $E_{k}^{L}$ before being redistributed between the ESS and the distribution grid. Therefore the prosumer energy balance is treated as a single aggregated subsystem that is represented by its corresponding energy flow value defined as

$E_{k}^{G L}=E_{k}^{G}+E_{k}^{L}, \quad k=1,2, \ldots$

Moreover, the prosumer energy balance $E_{k}^{G L}$ is assumed to be a known quantity read in real time by a smart meter. In each time slot, $E_{k}^{G L}$ must be redistributed between the main grid and the ESS in order to fulfill the following energy balance relation

$E_{k}^{S}+E_{k}^{N}+E_{k}^{G L}=0, \quad k=1,2, \ldots$

The MG energy flows are managed in real time by an EMS which evaluates the fraction of the prosumer energy balance $E_{k}^{G L}$ that must be exchanged with the ESS (i.e. $E_{k}^{S}$ ) in the $k$ th time slot. Therefore, the residual fraction of $E_{k}^{G L}$ (i.e. $E_{k}^{N}$ ) is exchanged with the main grid (see (5)).

As shown in Fig. 4, the inputs chosen for the EMS are the next prosumer energy balance $E_{k+1}^{G L}$, the present ESS SOC So $C_{k}$ and the next energy prices in sale $p_{k+1}^{\text {sell }}$ and purchasing $p_{k+1}^{\text {buy }}$.

\subsection{EMS synthesis}

The EMS previously introduced is synthesized by means of the GA-FIS paradigm discussed in Section 2.

The EMS is composed by two FISs, named FIS ${ }_{\text {sell }}$ and FIS buy as illustrated in Fig. 5. Both FISs have as input the actual SOC $S_{0} C_{k}$ and next time step energy balance, moreover $F I S_{\text {sell }}$ has the current sale energy price $p_{k}^{\text {sell }}$ as an additional input, whereas $F I S_{\text {buy }}$ is fed by the current purchasing energy prices $p_{k}^{\text {buy }}$ as illustrated in Fig. 5.

The two FISs work alternatively, the first in case of overproduction (i.e. $E_{k}^{G L}>0$ ) the second in case of over-demand (i.e. $E_{k}^{G L}<0$ ). More precisely, in case of over-demand (overproduction) the output of $F I S_{\text {buy }}\left(\right.$ FIS $\left.S_{\text {sell }}\right)$ represents the fraction of $E_{k}^{G L}$ to be discharged from (charged in) the ESS, whereas in case over-production (over-demand) the $F I S_{\text {buy }}\left(F I S_{\text {sell }}\right)$ output is assumed to be zero. Consequently, in order to estimate the amount of energy $E_{k+1}^{S}$ to exchange with the ESS during the next time slot the two FIS outputs are summed and then multiplied by $E_{k+1}^{G L}$, as shown in Fig. 5.

The GA optimization is used to tune the $\Theta_{M F}, \Theta_{R W}, \Theta_{R C}$ and $\Theta_{M H}$ subsets of both $F I S_{\text {buy }}$ and $F I S_{\text {sell }}$ according with a given OF $P$. In this approach the parameters of both the FISs belong to the same genetic variables set $\Omega$.

\section{Problem formulation and simulation settings}

\subsection{Problem formulation}

In this work the following four different optimization strategies of the proposed MG GA-FIS EMS have been considered:

- S1: An initial sub-optimization on $\Theta_{R C}, \Theta_{M F}$ and $\Theta_{R W}$ is applied. It is followed by a final optimization on $\Theta_{M H}$.

- S2: An initial sub-optimization on $\Theta_{R C}$ followed by a simultaneous sub-optimization on $\Theta_{M F}$ and $\Theta_{R W}$ is applied. Finally the hierarchical optimization on $\Theta_{M H}$ is performed.

- S3: The same optimization process described in $S 1$ is applied, except the Fuzzy input $E_{k+1}^{G L}$ (i.e. the energy balance) that is not considered in neither the $F I S_{\text {sell }}$ nor the $F I S_{\text {buy }}$. Therefore the two FISs have two Fuzzy inputs only.

- S4: The same optimization process described in $S 2$ is applied, except the Fuzzy input $E_{k+1}^{G L}$, as in the $S 3$ case.

It is clear that $S 2$ and $S 4$ are organized as a sequence of suboptimization processes unlike $S 1$ and $S 3$. For $S 2$ and $S 4$ the initial optimization on $\Theta_{R C}$ is motivated by the primary role of the Rule Base Inference System (i.e decision making strategy) with respect to the tuning of the MFs $\left(\Theta_{M F}\right)$ and rule weights $\left(\Theta_{R W}\right)$. In fact, $\Theta_{R C}$ has more influence on the decisional surface shape, since a mutation on a gene referred to $\Theta_{R C}$ is more relevant than a mutation on one gene associated to $\Theta_{M F}$ or $\Theta_{R W}$. In all cases the hierarchical optimization is executed downstream of the optimization process aiming to simplify the FISs structure and to delete those Fuzzy Sets which are not considered effective, necessary or not well tuned. The execution of $S 3$ and $S 4$ are considered to study the sensitivity of the proposed GA-FIS paradigm to the presence of the $E_{k+1}^{G L}$ Fuzzy input in terms of the generated profit and computational burden. The input simplification in $S 3$ and $S 4$ implies a reduction of variables in each subsets and consequently the complexity of the problem.

The adopted OF to be maximized is the total profit generated by the energy trading with the distribution grid in a given time period constituted by $N_{\text {slot }}$ time slots.

$P=\sum_{k=1}^{N_{\text {slot }}} P_{k}$

where, considering a TOU price policy, the income $P_{k}$ generated by the energy trade with the main grid at the $k$ th time slot is given by

$P_{k}= \begin{cases}E_{k}^{N} \cdot p_{k}^{\text {buy }} & \text { if } E_{k}^{N}>0 \\ E_{k}^{N} \cdot p_{k}^{\text {sell }} & \text { if } E_{k}^{N} \leq 0 .\end{cases}$

\subsection{Specific simulation scenario}

The specific MG considered in this work is composed by a PV generator, an aggregated load and an ESS. The PV generation and the aggregated load are characterized by $19.95 \mathrm{~kW}$ and $8 \mathrm{~kW}$ peak power, respectively. The ESS taken into consideration is $\mathrm{Li}$ ion SCiB battery pack produced by Toshiba (see Table 1). Data of aggregated power generation and consumption have been provided by areti S.p.A, the electric distribution utility that manages the power grid in the area of Rome. The aggregated loads and energy production time series consist of measurements made in the course of one year with a sampling rate of 15 minutes, on a real plant. However, for our test we have taken into account only the first 90 days, because of the high computational cost required to compute the optimal ideal solution, i.e. when having a perfect knowledge of all future samples (upper bound benchmark). In Fig. 6 are shown 7 days of the aggregated load and 


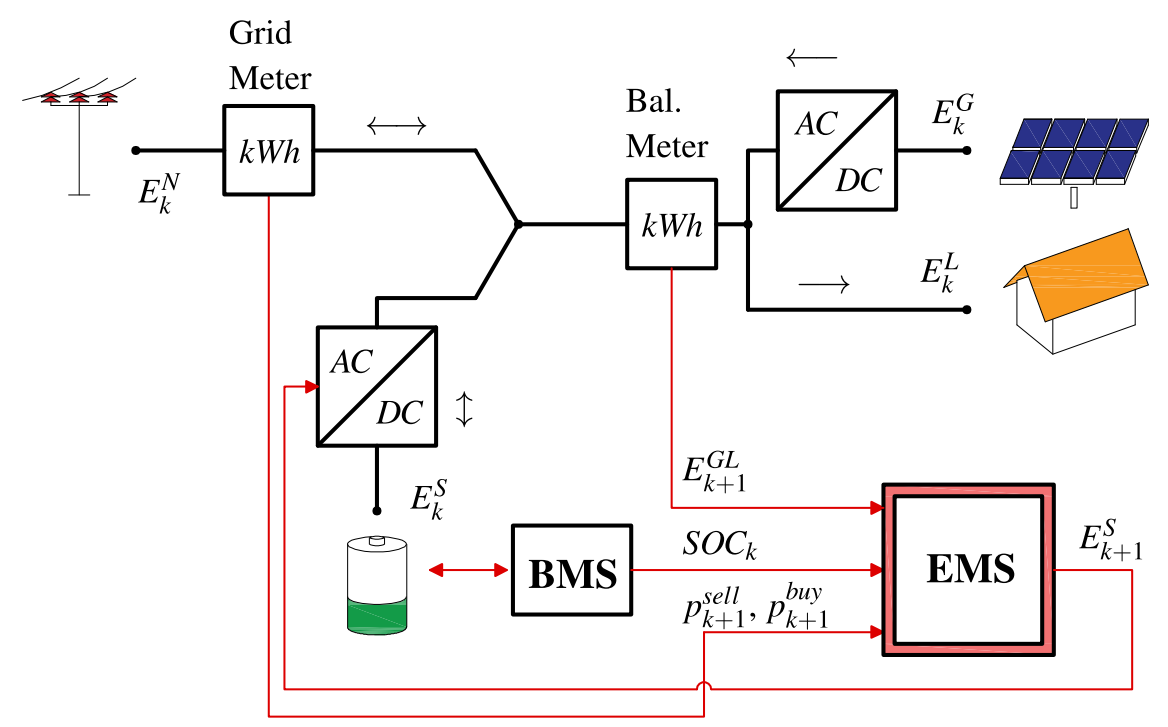

Fig. 4. MG architecture. Signal wires in red, power lines in black.

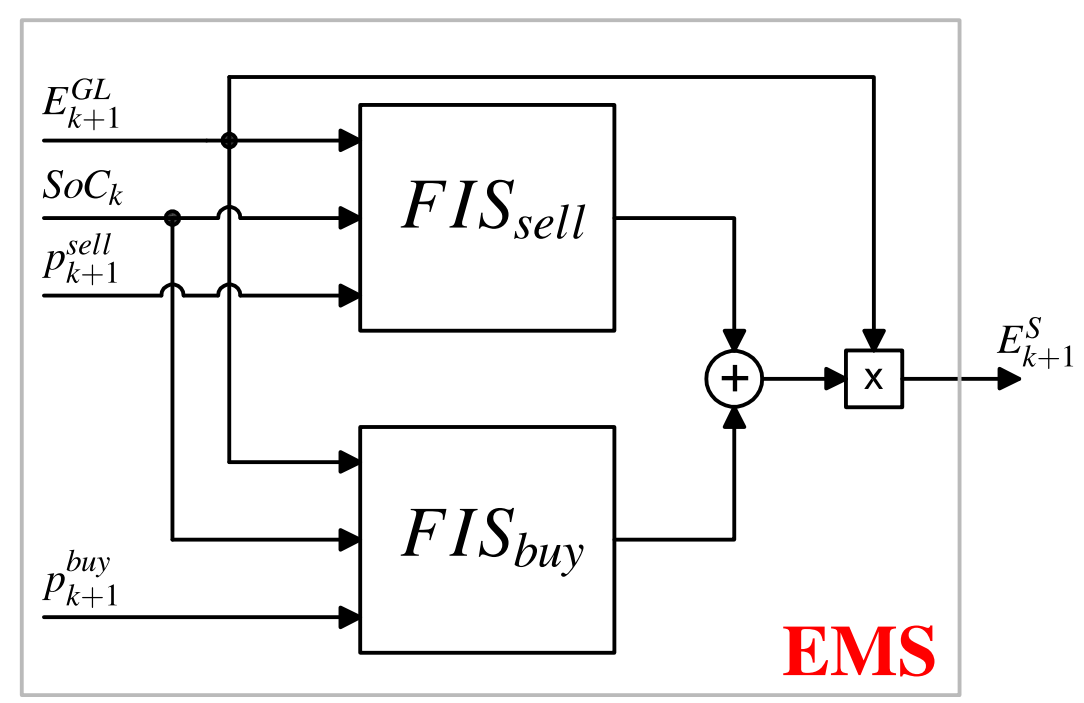

Fig. 5. EMS scheme.

Table 1

Toshiba SCiB module main characteristics.

\begin{tabular}{lllll}
\hline Capacity & Rated voltage & Current & Energy & Efficiency \\
\hline $80(\mathrm{Ah})$ & $300(\mathrm{~V})$ & 8 (C-Rate) & $24(\mathrm{kWh})$ & 1 (adim) \\
\hline
\end{tabular}

PV generation together with the energy price series. The energy prices are expressed in Monetary Unit per kWh $[M . U . / k W h]$. The overall prosumer dataset has been split in two disjoint sets taking the samples relative to the odd and the even days in order to build the Training Set (TR) and the Test Set (TS), respectively. The learning procedure is performed on the TR and the results are drawn on the TS. In Table 2 is detailed the prosumer dataset and its partitioning in TR and TS.

\subsection{GA-FIS settings}

In Table 3 are illustrated the GA parameter setting introduced in Section 2.3. The number of individuals $N_{\text {cross }}$ and $N_{\text {elite }}$ subject to the cross-over and the elitism operators are expressed in percentage of the size of the whole population having $N_{\text {ind }}$ individuals. The GA parameters are set in order to reach a balance of both the exploration and the exploitation capabilities. The $\Theta_{R C}$ initial population genes are randomly generated between their respective upper and lower bounds. The $\Theta_{M F}$ subset is set according to Fig. 1 (i.e. the MFs are equally spaced and symmetric with a $50 \%$ overlap). Each element of the subsets $\Theta_{R W}$ and $\Theta_{M H}$ is equal to one in order to give to all the rules the same degree of importance and to make active all the input Fuzzy Sets when the simulation starts. Summarizing, the individuals of $G_{0}$ differs from each other only for the genes associated with $\Theta_{R C}$. The $\Theta_{M F}$ subset is composed by $3 \cdot 5 \cdot 4 \cdot 2=120$ real variables (i.e. genes) where 3,5, 4 and 2 are the cardinality of the MFs, the Term Sets, the number of linguistic variables and the number of FISs (i.e. sell and buy FISs), respectively. The $\Theta_{R W}$ subset is composed by $5^{3} \cdot 2=250$ real valued genes $\in(0,1]$ interval. The $\Theta_{R C}$ subset has the same number of genes in $\Theta_{R W}$ but in this case they are discrete. $\Theta_{R C}$ genes assume values ranging from 1 to 5 


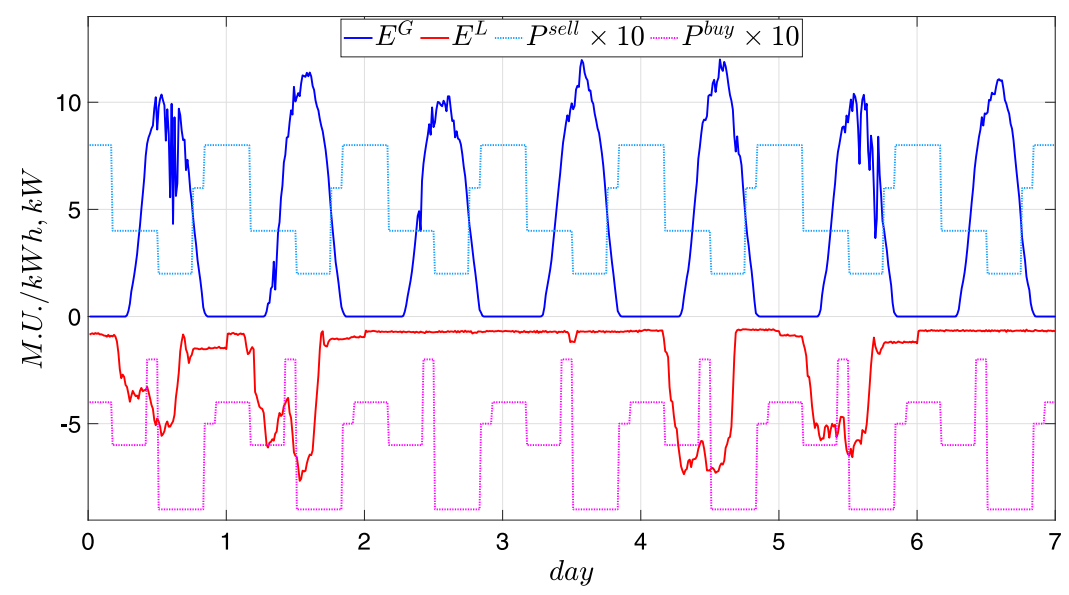

Fig. 6. MG power production and power demand profiles of the first 7 days of data together with the energy prices.

Table 2

Prosumer dataset description.

\begin{tabular}{llll}
\hline Dataset length & 8640 pattern & Time sample & 15 min \\
TS number of days & 45 (even days) & TR number of days & 45 (odd days) \\
Max. daily PV gen. & $92.82 \mathrm{kWh}$ & Max. PV power gen. & $11.84 \mathrm{~kW}$ \\
Max. daily energy dem. & $-13.93 \mathrm{kWh}$ & Max. power dem. & $-7.87 \mathrm{~kW}$ \\
\hline
\end{tabular}

Table 3

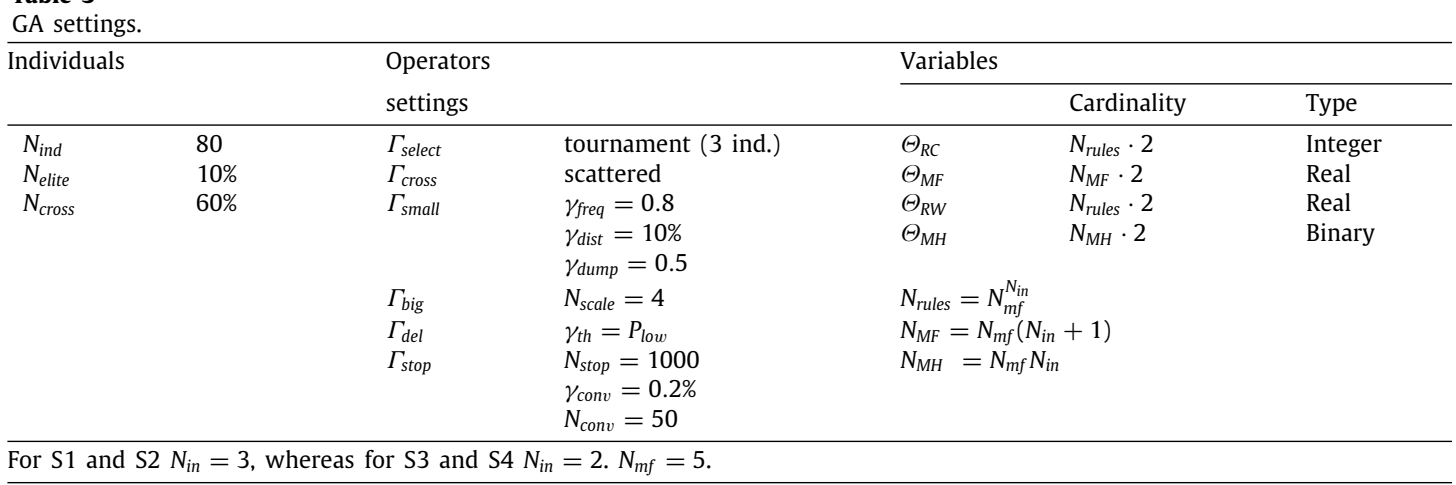

(i.e. the Term Set cardinality). $\Theta_{M H}$ is a binary set of size equal to the number of input Fuzzy Sets (i.e. $15 \cdot 2=30$ variables). Therefore, the overall $\Omega$ set owns 650 elements that represent the EMS parameters and the GA genes.

\section{Results}

\subsection{Profit lower and upper bounds}

In order to rate the results obtained by the four proposed GAFIS solutions, an upper and a lower bound have been estimated on the TS as benchmark solutions. Regarding the profit lower bound estimation, it has been considered as benchmark the solution $S_{\text {low }}$ obtained simulating the same MG devoid of the ESS. This choice seems to be reasonable because a solution having a profit lower than the one achieved without ESS is obviously detrimental. In this case in each time slot $E_{k}^{S}=0$, consequently, according with (5)

$$
E_{k}^{N}=-E_{k}^{G L} \quad k=1,2, . .
$$

the lower bound of the profit $P_{l o w}$ is obtained substituting (8) in (7).

For the profit upper bound performance estimation it has been chosen as benchmark the optimal solution $S_{u p p}$, considering known a priori all the TS time series, namely the power demand, the power generation and the energy prices. In this case it is possible to reformulate the optimization problem as a LP optimization problem that can be solved through the Simplex algorithm.

In order to apply the Simplex algorithm, for each time slot, $E_{k}^{G L}$ has been split in two parts representing the energy balance in over production and over demand defined as:

$E_{k}^{G L-}=\left\{\begin{array}{ll}E_{k}^{G L} & \text { if } E_{k}^{G L}<0 \\ 0 & \text { otherwise },\end{array} \quad E_{k}^{G L+}= \begin{cases}E_{k}^{G L} & \text { if } E_{k}^{G L} \geq 0 \\ 0 & \text { otherwise. }\end{cases}\right.$

According to Section 3, the problem is subject to the following constraints

$E_{k}^{G L}=E_{k}^{G L+}+E_{k}^{G L-}, \quad \forall k=1, \ldots, N$

$E_{k}^{S}=-\left(E_{k}^{S+}+E_{k}^{S-}\right), \quad \forall k=1, \ldots, N$

$E_{k}^{N-}=E_{k}^{G L-}-E_{k}^{S-}, \quad \forall k=1, \ldots, N$

$E_{k}^{N+}=E_{k}^{G L+}-E_{k}^{S+}, \quad \forall k=1, \ldots, N$

$0 \geq E_{k}^{S-} \geq E_{k}^{G L-}, \quad \forall k=1, \ldots, N$

$0 \leq E_{k}^{S+} \leq E_{k}^{G L+}, \quad \forall k=1, \ldots, N$

$E^{\text {dch-max }} \leq-E_{k}^{S} \leq E^{\text {ch-max }}, \quad \forall k=1, \ldots, N$ 
Table 4

ENS prediction performances.

\begin{tabular}{|c|c|c|c|c|c|c|}
\hline \multicolumn{7}{|c|}{ Prediction on PV Generation- $E^{G}$} \\
\hline t-slot & $1-8$ & $9-16$ & $17-24$ & $25-32$ & $33-40$ & $41-48$ \\
\hline RMSE & $0.06 \pm 0.01$ & $0.06 \pm 0.00$ & $0.06 \pm 0.00$ & $0.06 \pm 0.00$ & $0.06 \pm 0.00$ & $0.06 \pm 0.00$ \\
\hline t-slot & $49-56$ & $57-64$ & $65-72$ & $73-80$ & $81-88$ & $89-96$ \\
\hline RMSE & $0.07 \pm 0.00$ & $0.07 \pm 0.00$ & $0.07 \pm 0.00$ & $0.07 \pm 0.01$ & $0.07 \pm 0.01$ & $0.07 \pm 0.00$ \\
\hline \multicolumn{7}{|c|}{ Prediction on Prosumer Energy Demand- $E^{L}$} \\
\hline t-slot & $1-8$ & $9-16$ & $17-24$ & $25-32$ & $33-40$ & $41-48$ \\
\hline RMSE & $0.08 \pm 0.03$ & $0.12 \pm 0.02$ & $0.12 \pm 0.01$ & $0.13 \pm 0.01$ & $0.15 \pm 0.01$ & $0.16 \pm 0.02$ \\
\hline t-slot & $49-56$ & $57-64$ & $65-72$ & $73-80$ & $81-88$ & $89-96$ \\
\hline RMSE & $0.17 \pm 0.02$ & $0.18 \pm 0.02$ & $0.20 \pm 0.03$ & $0.18 \pm 0.01$ & $0.17 \pm 0.01$ & $0.17 \pm 0.02$ \\
\hline
\end{tabular}

$E^{\text {min }}-E^{S 0} \leq \sum_{i=0}^{i=k}-E_{i}^{S} \leq E^{\text {max }}-E^{S 0}, \quad \forall k=1, \ldots, N$

where $E_{k}^{S+}$ and $E_{k}^{S-}$ are different from zero when the ESS is charged and discharged, respectively. In (17) the energy of the ESS is constrained between its upper and lower limits. $E^{S 0}$ is the initial energy ESS state.

The LP optimal OF value is used to compare the results given by the designed FIS EMSs. Therefore, taking into consideration (6) and (7), it is equal to

$P_{\text {upp }}=\sum_{k=0}^{k=N}\left(E_{k}^{N-} \cdot p_{k}^{\text {buy }}+E_{k}^{N+} \cdot p_{k}^{\text {sell }}\right)$.

\subsection{A prediction based benchmark solution formulation}

The application of prediction algorithms on the prosumer can be used by the EMS for recursively evaluate and apply the first element of a reference optimal solution time series evaluated by a suitable optimization algorithm. It is clear that the application of this strategy, named RTH, relies on the prediction accuracy and the time horizon length which is usually set to one or even two days as in [34].

In this work the model adopted, named $S_{R T H}$ is defined by two ESN based prediction systems in charge to predict the future PV generation and energy demand of the prosumer, respectively. The prediction model used is the same proposed in [43], which is based on the ESN described in [68-70]. In this model each prediction system is composed by a number of ESNs equal to the number of time frames covering the prediction time horizon. In particular, each ESN is in charge to predict the future energy generation (demand) at a given number of time frames ahead. Therefore, considering a prediction time horizon of one day and two distinct energy systems to predict, the number of ESNs to train is $2 \times 96$.

As explained in [43], the ESN inputs are the current energy production (demand) of the assigned energy system, the current hour and a raw estimation of the daily energy generation (demand). The inputs and the output are defined in $[0 ; 1]$. In particular, the energy signals are normalized between their respective maximum (absolute) value registered on the TR. The ESNs are synthesized through a GA which defines the reservoir and the input layer [70] considering the same procedure proposed in [43]. The OF is formulated in order to consider both the prediction accuracy and complexity

$f=0.99 \cdot \Phi+10^{-1} \cdot \Gamma+10^{-4} \cdot \Psi$

where $\Phi$ is the Root Mean Square Error (RMSE) with respect to the signal to predict, $\Gamma$ is the sum of the (optimized) output weights divided by its own length and $\Psi$ is the reservoir size normalized by the respective lower and upper bound.

It is important to note that the ESN synthesis procedure by GA requires a TR and a Validation Set. Indeed, given a GA individual which defines the ESN reservoir and the input layer, the output layer is calculated on the TR by means of a mean square regression. During genetic optimization, the fitness function (19) is evaluated on the Validation Set. For generating the Validation Set, the TR defined in Section 4.2 has been split again in two subsets named Training Subset and Validation Subset. In particular, 30 (randomly chosen) days of the TR have been assigned to the Training Subset, whereas the other 15 days are assigned to the Validation Subset (see Table 2). The GA has been set with the same operators introduced in Section 4.3 with the exception of $N_{\text {stop }}=50$ considering as OF the expression (19).

In Table 4 are shown the RMSE mean values ( \pm standard deviation) of the generated ESNs which are grouped per assigned time slot and time series to predict.

\subsection{Results discussion}

In this section are illustrated the results of the solution introduced in Sections 3, 5.1 and 5.2. All tests have been implemented in Matlab ${ }^{\circledR} R 2019 a$ on a workstation equipped with two Intel ${ }^{\circledR}$ Xeon ${ }^{\circledR}$ 6-cores CPUs at $2.40 \mathrm{GHz}$. The following libraries have been used: CVX [71,72] for the evaluation of the optimal energy distribution strategy over a give time horizon, the ESN model proposed by Jaeger et al. ${ }^{1}$ for the prediction systems design and finally the Matlab ${ }^{\circledR}$ built-in Fuzzy Logic Toolbox for FISs implementation.

All the simulations results are reported in Table 5 . There, the solutions $S 1, S 2, S 3$ and $S 4$ are compared with the upper and lower benchmark solutions $S_{\text {low }}, S_{\text {upp }}$ and two variants of the RTH strategy solution (see Section 5.2) $S_{R T H-E S N}$ and $S_{R T H-i d e a l}$. The first refers to a RTH EMS supported by two ESN-prediction systems with performance illustrated in Table 4, whereas the second considers a zero-error prediction. $S_{R T H-i d e a l}$ is therefore the $S_{R T H-E S N}$ upper limit. It is important to note that though both in $S_{R T H-i d e a l}$ and $S_{\text {upp }}$ is assumed a (perfect) a priori knowledge of the future time series, in $S_{R T H-i d e a l}$ it is restricted to a time horizon of one day, whereas in $S_{u p p}$ is extended to the overall TS.

Regarding the Fuzzy EMSs simulation $S 1, S 2, S 3$ and $S 4$, tests have been repeated 5 times with different randomly generated initial populations that differs only on $\Theta_{R C}$ as explained in Section 4.3. The values reported in Table 5 represent the average values and the standard deviation (in brackets). Each suboptimization step of each simulation is analysed by simulating their respective best individual on the TS. In Table 5 for each sub-optimization step the remainder of subsets to be optimized are indicated with the superscript ' 0 ', whereas those that were already optimized are denoted with the superscript ${ }^{*}$ ',

The simulation performances are rated in terms of profit (see (6)) and number of executed generations. These values are used to estimate the effectiveness and efficiency of the proposed EMS optimization paradigm, respectively.

1 http://minds.jacobs-university.de/research/esnresearch/. 
Table 5

Optimized EMSs TS results.

\begin{tabular}{|c|c|c|c|c|c|c|c|c|c|c|}
\hline \multirow[t]{2}{*}{ Simulation } & \multirow[t]{2}{*}{ Initial population } & \multirow{2}{*}{$\begin{array}{l}\text { Optimized } \\
\text { parameters }\end{array}$} & \multirow{2}{*}{$\begin{array}{l}\text { TS profit } \\
\text { [MU] }\end{array}$} & \multicolumn{2}{|c|}{ Energy Sold } & \multicolumn{2}{|c|}{ Energy Bought } & \multirow{2}{*}{$\begin{array}{l}\text { ESS expl. } \\
{[\mathrm{kWh}]}\end{array}$} & \multirow{2}{*}{$\begin{array}{l}\text { \# Deleted fuzzy } \\
\text { set [adim] }\end{array}$} & \multirow{2}{*}{$\begin{array}{l}\text { \# Generations } \\
\text { [adim] }\end{array}$} \\
\hline & & & & [kWh] & {$[\mathrm{MU}]$} & [kWh] & [MU] & & & \\
\hline \multirow{2}{*}{$\begin{array}{l}S 1-a \\
S 1-b\end{array}$} & \multirow{2}{*}{$\begin{array}{l}\Theta_{R C}^{0}, \Theta_{M F}^{0}, \Theta_{R W}^{0}, \Theta_{M H}^{0} \\
\Theta_{R C}^{*}, \Theta_{M F}^{*}, \Theta_{R W}^{*}, \Theta_{M H}^{0}\end{array}$} & $\Theta_{R C}, \Theta_{M F}, \Theta_{R W}$ & $102.3(1.6)$ & $961(14)$ & $283(3)$ & $349(12)$ & $174(4)$ & $1286(25)$ & & $650(211)+$ \\
\hline & & $\Theta_{M H}$ & $104.2(1)$ & $957(12)$ & $283(2)$ & $347(12)$ & $172(3)$ & $1290(24)$ & $10(2)$ & $\begin{array}{l}98(40)= \\
748(251)\end{array}$ \\
\hline \multirow{3}{*}{$\begin{array}{l}S 2-a \\
S 2-b \\
S 2-c\end{array}$} & \multirow{3}{*}{$\begin{array}{l}\Theta_{R C}^{0}, \Theta_{M F}^{0}, \Theta_{R W}^{0}, \Theta_{M H}^{0} \\
\Theta_{R C}^{*}, \Theta_{M F}^{0}, \Theta_{R W}^{0}, \Theta_{M H}^{0} \\
\Theta_{R C}^{*}, \Theta_{M F}^{*}, \Theta_{R W}^{*}, \Theta_{M H}^{0}\end{array}$} & $\Theta_{R C}$ & $96.2(2.6)$ & $945(10)$ & $278(2)$ & $334(10)$ & $175(2)$ & $1315(20)$ & & $748(368)+$ \\
\hline & & $\Theta_{R W}, \Theta_{M F}$ & $105.4(0.8)$ & $944(10)$ & $280(2)$ & $332(10)$ & $167(3)$ & 1092(20) & & $346(110)+$ \\
\hline & & $\Theta_{M H}$ & $105.8(0.5)$ & $943(10)$ & $280(2)$ & $332(10)$ & $166(2)$ & $1320(20)$ & $5(4)$ & $\begin{array}{l}75(40)= \\
1170(518)\end{array}$ \\
\hline \multirow{2}{*}{$\begin{array}{l}S 3-a \\
S 3-b\end{array}$} & \multirow{2}{*}{$\begin{array}{c}\Theta_{R C}^{0}, \Theta_{M F}^{0}, \Theta_{R W}^{0}, \Theta_{M H}^{0} \\
\Theta_{M H}^{0}, \Theta_{R C}^{*}, \Theta_{M F}^{*}, \Theta_{R W}^{*}\end{array}$} & $\Theta_{R C}, \Theta_{M F}, \Theta_{R W}$ & $103.7(0.8)$ & $967(13)$ & $285(3)$ & $356(13)$ & $174(3)$ & $1272(26)$ & & $512(188)+$ \\
\hline & & $\Theta_{M H}$ & $104.0(0.6)$ & $975(6)$ & $286(1)$ & $364(6)$ & $175(2)$ & $1256(13)$ & $9(1)$ & $\begin{array}{l}68(11)= \\
581(198)\end{array}$ \\
\hline \multirow{3}{*}{$\begin{array}{l}S 4-a \\
S 4-b \\
S 4-c\end{array}$} & \multirow{3}{*}{$\begin{array}{l}\Theta_{R C}^{0}, \Theta_{M F}^{0}, \Theta_{R W}^{0}, \Theta_{M H}^{0} \\
\Theta_{R C}^{*}, \Theta_{M F}^{0}, \Theta_{R W}^{0}, \Theta_{M H}^{0} \\
\Theta_{R C}^{*}, \Theta_{R W}^{*}, \Theta_{R C}^{*}, \Theta_{M H}^{0}\end{array}$} & $\Theta_{R C}$ & $102.2(0.5)$ & $969(1)$ & $285(1)$ & $359(1)$ & $176(1)$ & $1267(3)$ & & $277(85)+$ \\
\hline & & $\Theta_{R W}, \Theta_{M F}$ & $104.2(0.7)$ & $971(3)$ & $286(1)$ & $363(7)$ & $174(1)$ & $1045(5)$ & & $378(223)+$ \\
\hline & & $\Theta_{M H}$ & $104.3(0.6)$ & $971(3)$ & $286(1)$ & $360(3)$ & $174(6)$ & $1263(4)$ & $6(4)$ & $\begin{array}{l}52(4)= \\
707(312)\end{array}$ \\
\hline$S_{\text {upp }}$ & - & - & 135.1 & 901 & 270 & 289 & 135 & 1405 & - & - \\
\hline$S_{\text {low }}$ & - & - & -144.6 & 1599 & 411 & 997 & 556 & 0 & - & - \\
\hline$S_{\text {RTH-ESN }}$ & - & - & 102.4 & 971 & 281 & 361 & 182 & 1277 & - & - \\
\hline$S_{\text {RTH-ideal }}$ & - & - & 119.3 & 936 & 271 & 326 & 156 & 1357 & - & - \\
\hline
\end{tabular}


Moreover, in Table 5 are shown the overall energy sold and bought expressed in terms of both M.U. and $k W h$, the total ESS exploitation that is evaluated as the absolute sum of $E_{K}^{S}$ extended to the overall simulation (i.e. $\sum_{k}\left|E_{k}^{S}\right|$ ), and finally the number of Fuzzy Sets deleted in each hierarchical optimization procedure.

Simulations which privileges an EMS optimization of the $\Omega$ parameters developed in more stages (i.e. S2 and S4) present always greater profits with respect to their corresponding optimizations procedures, namely $S 1$ and $S 3$, respectively. However, they are characterized by an increase in the overall efficiency, that is affected by a higher standard deviation as well.

The simultaneous approach followed in $S 1$ and $S 3$ shows a greater number of deleted Fuzzy Sets, on average almost double with respect to the other two procedures (i.e. 9.5 vs 5.5), thanks to the hierarchical optimization. In fact, when the subsets $\Theta_{R C}, \Theta_{M F}, \Theta_{R W}$ are simultaneously optimized, the GA shows a poor ability in tuning the FIS nearby optimum or sub-optimum values. It can be noted that the optimization on the $\Theta_{R C}$ subset on $S 4-a$ shows a profit lower by $3.5 \%$ with respect to $S 2-c$, but much higher than the profit achieved by $S 2-a$ (i.e. 102.2 Vs 96.2 M.U.). However, looking at the executed number of generations and the profit standard deviation, solution $S 4-a$ produces the best performances in terms of efficiency and robustness.

On the contrary, simulation $S 2-a$ shows that the optimization of the $\Theta_{R C}$ subset, when the prosumer energy balance is considered as an additional input, gives the worst results in terms of both efficiency and effectiveness. In fact, in this case the GA presents difficulties in finding a good solution in a larger space. However, the subsequent optimizations performed on the remaining subsets bring the profit to higher values, more precisely to the best one. The Fuzzy input energy balance increases the average profit less than $1 \%$ despite a decrease of their robustness and efficiency (i.e. higher \# Generations, standard deviations on the profit and standard deviations on \# Generations).

As it is shown in Table 5, all the Fuzzy EMS solutions present a profit within the range $86 \div 90 \%$ with respect to $P_{\text {upp }}-P_{\text {low }}$ interval, namely about $10 \%$ close to the $S_{\text {upp }}$ solution. Looking at the overall energy sold and the overall energy bought, all the solutions show values close to the $S_{u p p}$ solution that produce a reduction of the overall energy exchanged to the network greater than $50 \%$ of the overall energy exchanged by solution $S_{\text {low }}$. In most cases the hierarchical optimization, other than improving the profits, reduce the EMS number of rules by more than half. Comparing with the RTH solutions, the profit of $S_{R T H-i d e a l}$ is very close to $P_{\text {upp }}$ (just 5\% below). However, $S_{R T H-E S N}$, which performs with uncertainties on the prediction up to the $7 \%$ on the PV generation and $20 \%$ on the energy demand (see Table 4 in Section 5.2), owns a profit similar to the Fuzzy EMS.

\section{Conclusion}

In this work we compared four different optimization strategies of a MG EMS defined by two FISs in order to redistribute in an intelligent way the prosumer energy balance. The MG is supposed to be equipped with an ESS and to be connected to the main grid. The optimization, performed off line on TR data through a GA, aims at minimizing the profit generated by the energy trading with the main grid during a given time period on a TS, exploiting energy buffering in ESS.

The EMS optimization parameters were grouped in four subsets representing the rule consequents association, the MFs shape, the rule weights and the deactivation of input Fuzzy Sets. This latter has the role to delete such input Fuzzy Sets that were inefficiently arranged and therefore to simplify the EMS rule base.

The adopted strategies differ in the order in which the optimization of such parameter subsets is applied. In particular, one strategy privileges a simultaneous optimization of the parameters relative to the rule consequents association, the MFs shape and the rule weights. The second strategy first define the FISs rule base inference system (i.e. the rule consequents association) and successively tunes simultaneously the MFs shape and the rule weights. Moreover, the simulations are repeated without considering the prosumer energy balance as Fuzzy Input in order to study how it affects the EMS performances, possibly improving the interpretability of the Fuzzy rule base.

TS results show that performances related to the profit of optimized EMSs are close to $90 \%$ of the optimal performance (upper bound). Moreover, the hierarchical optimization helps to reduce the number of rules by more than half, allowing a more interpretable EMS, especially in case where the FISs are featured by two inputs. It this case the EMS rule base system can be reduced up to less than 25 rules. Therefore, it proved to be very effective in improving the overall interpretability of the solution found, yielding a lighter rule base, better suited for running, at run time, on low cost microcontrollers. An ESN based RTH strategy EMS is used for a deeper comparison of the results. It is worth to note that, although the RTH EMS shows comparable results on profit, this system is characterized by a much higher computational cost at training stage, as well as a higher structural complexity of the trained model, due to the presence of prediction ESN models. Results show that FIS based EMSs have similar performances with respect to the RTH-based strategy in terms of the overall profit, with a remarkable advantage in terms of computational cost at run time.

\section{Declaration of competing interest}

No author associated with this paper has disclosed any potential or pertinent conflicts which may be perceived to have impending conflict with this work. For full disclosure statements refer to https://doi.org/10.1016/j.asoc.2019.105903.

\section{References}

[1] A. Ali, W. Li, R. Hussain, X. He, B.W. Williams, A.H. Memon, Overview of current microgrid policies, incentives and barriers in the european union, United States and China, Sustainability 9 (7) (2017).

[2] M. Amer, A. Naaman, N.K. M'Sirdi, A.M. El-Zonkoly, Smart home energy management systems survey, in: International Conference on Renewable Energies for Developing Countries 2014, 2014, pp. 167-173, http://dx.doi. org/10.1109/REDEC.2014.7038551.

[3] A. Izadian, N. Girrens, P. Khayyer, Renewable energy policies: A brief review of the latest U.S. and E.U. policies, IEEE Ind. Electron. Mag. 7 (3) (2013) 21-34, http://dx.doi.org/10.1109/MIE.2013.2269701.

[4] H. Wirth, Recent facts about photovoltaics in Germany, 2018, https: //www.ise.fraunhofer.de/content/dam/ise/en/documents/publications/ studies/recent-facts-about-photovoltaics-in-germany.pdf.

[5] D. Meyer, J. Wang, Integrating ultra-fast charging stations within the power grids of smart cities: a review, IET Smart Grid 1 (1) (2018) 3-10, http://dx.doi.org/10.1049/iet-stg.2018.0006.

[6] D. Steen, L.A. Tuan, Fast charging of electric buses in distribution systems, in: 2017 IEEE Manchester PowerTech, 2017, pp. 1-6.

[7] A. Zegers, H. Brunner, TSO-DSO interaction: An Overview of current interaction between transmission and distribution system operators and an assessment of their cooperation in Smart Grids, International Smart Grid Action Network (ISAGN) Discussion Paper Annex, 6, 2014.

[8] R. Deng, Z. Yang, M.Y. Chow, J. Chen, A survey on demand response in smart grids: Mathematical models and approaches, IEEE Trans. Ind. Inf. 11 (3) (2015) 570-582, http://dx.doi.org/10.1109/TII.2015.2414719.

[9] S. Lavrijssen, A. Carrillo Parra, Radical prosumer innovations in the electricity sector and the impact on prosumer regulation, Sustainability 9 (2017) 1207, http://dx.doi.org/10.3390/su9071207.

[10] D.T. Ton, M.A. Smith, The U.S. department of energy's microgrid initiative, Electr. J. 25 (8) (2012) 84-94.

[11] R.H. Lasseter, P. Paigi, Microgrid: a conceptual solution, in: 2004 IEEE 35th Annual Power Electronics Specialists Conference (IEEE Cat. No.04CH37551), Vol. 6, 2004, pp. 4285-4290, http://dx.doi.org/10.1109/PESC.2004.1354758.

[12] About microgrids, 2018, online. 
[13] T. Dragicevic, J.C. Vasquez, J.M. Guerrero, D. Skrlec, Advanced LVDC electrical power architectures and microgrids: A step toward a new generation of power distribution networks, IEEE Electrif. Mag. 2 (1) (2014) 54-65, http://dx.doi.org/10.1109/MELE.2013.2297033.

[14] J. Shen, A. Khaligh, A supervisory energy management control strategy in a battery/ultracapacitor hybrid energy storage system, IEEE Trans. Transp. Electr. 1 (3) (2015) 223-231, http://dx.doi.org/10.1109/TTE.2015.2464690.

[15] S.H. Mahyiddin, M.R. Mohamed, Z. Mustaffa, A.C. Khor, M.H. Sulaiman, H. Ahmad, S.A. Rahman, Fuzzy Logic energy management system of series hybrid electric vehicle, in: 4th IET Clean Energy and Technology Conference (CEAT 2016), 2016, pp. 1-6, http://dx.doi.org/10.1049/cp.2016.1267.

[16] R. Mohanty, A.K. Pradhan, Protection of DC and hybrid AC-dc microgrids with ring configuration, in: 2017 7th International Conference on Power Systems (ICPS), 2017, pp. 607-612, http://dx.doi.org/10.1109/ICPES.2017. 8387365.

[17] H. Erteza Gelani, F. Dastgeer, K. Siraj, M. Nasir, K.A.K. Niazi, Y. Yang, Efficiency comparison of AC and DC distribution networks for modern residential localities, Appl. Sci. 9 (2019) http://dx.doi.org/10.3390/ app9030582.

[18] B.T. Patterson, Dc, come home: DC microgrids and the birth of the "enernet", IEEE Power Energy Mag. 10 (6) (2012) 60-69, http://dx.doi.org/ 10.1109/MPE.2012.2212610.

[19] E. Rodriguez-Diaz, E.J. Palacios-Garcia, A. Anvari-Moghaddam, J.C. Vasquez, J.M. Guerrero, Real-time energy management system for a hybrid AC/DC residential microgrid, in: 2017 IEEE Second International Conference on DC Microgrids, ICDCM, 2017, pp. 256-261, http://dx.doi.org/10.1109/ICDCM. 2017.8001053.

[20] A. Gupta, S. Doolla, K. Chatterjee, Hybrid AC-DC microgrid: Systematic evaluation of control strategies, IEEE Trans. Smart Grid 9 (4) (2018) 3830-3843, http://dx.doi.org/10.1109/TSG.2017.2727344.

[21] D.E. Olivares, A. Mehrizi-Sani, A.H. Etemadi, C.A. Caizares, R. Iravani, M. Kazerani, A.H. Hajimiragha, O. Gomis-Bellmunt, M. Saeedifard, R. PalmaBehnke, G.A. Jiménez-Estévez, N.D. Hatziargyriou, Trends in microgrid control, IEEE Trans. Smart Grid 5 (4) (2014) 1905-1919, http://dx.doi.org/ 10.1109/TSG.2013.2295514.

[22] M. Zachar, P. Daoutidis, Microgrid/macrogrid energy exchange: A novel market structure and stochastic scheduling, IEEE Trans. Smart Grid 8 (1) (2017) 178-189, http://dx.doi.org/10.1109/TSG.2016.2600487.

[23] S. Parhizi, H. Lotfi, A. Khodaei, S. Bahramirad, State of the art in research on microgrids: A review, IEEE Access 3 (2015) 890-925

[24] D. Zhang, X. Han, C. Deng, Review on the research and practice of deep learning and reinforcement learning in smart grids, CSEE J. Power Energy Syst. 4 (3) (2018) 362-370, http://dx.doi.org/10.17775/CSEEJPES. 2018.00520.

[25] E. Foruzan, L. Soh, S. Asgarpoor, Reinforcement learning approach for optimal distributed energy management in a microgrid, IEEE Trans. Power Syst. 33 (5) (2018) 5749-5758, http://dx.doi.org/10.1109/TPWRS.2018.2823641.

[26] G.K. Venayagamoorthy, R.K. Sharma, P.K. Gautam, A. Ahmadi, Dynamic energy management system for a smart microgrid, IEEE Trans. Neural Netw. Learn. Syst. 27 (8) (2016) 1643-1656, http://dx.doi.org/10.1109/ TNNLS.2016.2514358.

[27] P. Mancarella, G. Chicco, Real-time demand response from energy shifting in distributed multi-generation, IEEE Trans. Smart Grid 4 (4) (2013) 1928-1938, http://dx.doi.org/10.1109/TSG.2013.2258413.

[28] O. Sundstrom, L. Guzzella, A generic dynamic programming matlab function, in: 2009 IEEE Control Applications, (CCA) Intelligent Control, (ISIC), 2009, pp. 1625-1630.

[29] A. Scalfati, D. Iannuzzi, M. Fantauzzi, M. Roscia, Optimal sizing of distributed energy resources in smart microgrids: A mixed integer linear programming formulation, in: 2017 IEEE 6th International Conference on Renewable Energy Research and Applications, ICRERA, 2017, pp. 568-573.

[30] S. Leonori, A. Martino, F.M.F. Mascioli, A. Rizzi, ANFIS Microgrid energy management system synthesis by hyperplane clustering supported by neurofuzzy min-max classifier, IEEE Trans. Emerg. Top. Comput. Intell. 3 (3) (2019) 193-204, http://dx.doi.org/10.1109/TETCI.2018.2880815.

[31] A.C. Luna, N.L. Diaz, M. Graells, J.C. Vasquez, J.M. Guerrero, Mixed-integerlinear-programming-based energy management system for hybrid PVwind-battery microgrids: Modeling, design, and experimental verification, IEEE Trans. Power Electron. 32 (4) (2017) 2769-2783.

[32] M.F. Zia, E. Elbouchikhi, M. Benbouzid, Microgrids energy management systems: A critical review on methods, solutions, and prospects, Appl. Energy 222 (2018) 1033-1055, http://dx.doi.org/10.1016/j.apenergy.2018.04.103, http://www.sciencedirect.com/science/article/pii/S0306261918306676.

[33] J. Silvente, G.M. Kopanos, E.N. Pistikopoulos, A. Espua, A rolling horizon optimization framework for the simultaneous energy supply and demand planning in microgrids, Appl. Energy 155 (2015) 485-501, http:/ dx.doi.org/10.1016/j.apenergy.2015.05.090, http://www.sciencedirect.com/ science/article/pii/S0306261915007230.
[34] R. Palma-Behnke, C. Benavides, F. Lanas, B. Severino, L. Reyes, J. Llanos, D. Sáez, A microgrid energy management system based on the rolling horizon strategy, IEEE Trans. Smart Grid 4 (2) (2013) 996-1006, http: //dx.doi.org/10.1109/TSG.2012.2231440.

[35] Y. Lan, X. Guan, J. Wu, Rollout strategies for real-time multi-energy scheduling in microgrid with storage system, IET Gener. Transm. Distrib. 10 (3) (2016) 688-696, http://dx.doi.org/10.1049/iet-gtd.2015.0426.

[36] S. Leonori, M. Paschero, A. Rizzi, F.M.F. Mascioli, An optimized microgrid energy management system based on FIS-MO-ga paradigm, in: 2017 IEEE International Conference on Fuzzy Systems (FUZZ-IEEE), 2017, pp. 1-6, http://dx.doi.org/10.1109/FUZZ-IEEE.2017.8015438.

[37] C. Voyant, G. Notton, S. Kalogirou, M.-L. Nivet, C. Paoli, F. Motte, A. Fouilloy, Machine learning methods for solar radiation forecasting: A review, Renew. Energy 105 (2017) 569-582, http://dx.doi.org/10.1016/ j.renene.2016.12.095, http://www.sciencedirect.com/science/article/pii/ S0960148116311648.

[38] A. Mosavi, M. Salimi, S.F. Ardabili, T. Rabczuk, S. Shamshirband, A. Varkonyi-Koczy, State of the art of machine learning models in energy systems, a systematic review, Energies 12 (7) (2019) 1301-1345, http: //dx.doi.org/10.3390/en12071301, https://eprints.qut.edu.au/128290/.

[39] W. Kong, Z.Y. Dong, Y. Jia, D.J. Hill, Y. Xu, Y. Zhang, Short-term residential load forecasting based on LSTM recurrent neural network, IEEE Trans. Smart Grid 10 (1) (2019) 841-851, http://dx.doi.org/10.1109/TSG.2017. 2753802.

[40] R. Jiao, T. Zhang, Y. Jiang, H. He, Short-term non-residential load forecasting based on multiple sequences LSTM recurrent neural network, IEEE Access 6 (2018) 59438-59448, http://dx.doi.org/10.1109/ACCESS.2018.2873712.

[41] T. Kim, S. Cho, Particle swarm optimization-based CNN-LSTM networks for forecasting energy consumption, in: 2019 IEEE Congress on Evolutionary Computation (CEC), 2019, pp. 1510-1516, http://dx.doi.org/10.1109/CEC. 2019.8789968.

[42] F.M. Bianchi, E. Maiorino, M.C. Kampffmeyer, A. Rizzi, R. Jenssen, An overview and comparative analysis of recurrent neural networks for short term load forecasting, 2017, arXiv preprint arXiv:1705.04378.

[43] S. Leonori, A. Rizzi, M. Paschero, F.M.F. Mascioli, Microgrid energy management by ANFIS supported by an ESN based prediction algorithm, in: 2018 International Joint Conference on Neural Networks, IJCNN, 2018, pp. 1-8, http://dx.doi.org/10.1109/IJCNN.2018.8489018.

[44] A. Rosato, R. Altilio, R. Araneo, M. Panella, A smart grid in ponza island: Battery energy storage management by echo state neural network, in: 2018 IEEE International Conference on Environment and Electrical Engineering and 2018 IEEE Industrial and Commercial Power Systems Europe (EEEIC/I CPS Europe), 2018, pp. 1-4, http://dx.doi.org/10.1109/EEEIC.2018.8493820.

[45] A. Rosato, R. Altilio, R. Araneo, M. Panella, Prediction in photovoltaic power by neural networks, Energies 10 (7) (2017).

[46] A. Zaaoumi, A. Bah, M. Alaoui, A. Mechaqrane, M. Berrehili, Application of artificial neural networks and adaptive neuro-fuzzy inference system to estimate the energy generation of a solar power plant in ain benimathar (Morocco), in: 2018 10th International Conference on Electronics, Computers and Artificial Intelligence, ECAI, 2018, pp. 1-6, http://dx.doi. org/10.1109/ECAI.2018.8679015.

[47] J. Liu, H. Chen, W. Zhang, B. Yurkovich, G. Rizzoni, Energy management problems under uncertainties for grid-connected microgrids: a chance constrained programming approach, IEEE Trans. Smart Grid PP (99) (2016) 1-1. http://dx.doi.org/10.1109/TSG.2016.2531004.

[48] A. Askarzadeh, A memory-based genetic algorithm for optimization of power generation in a microgrid, IEEE Trans. Sustain. Energy 9 (3) (2018) 1081-1089, http://dx.doi.org/10.1109/TSTE.2017.2765483.

[49] F. Lezama, E. Sucar, J. Soares, Z. Vale, E. Munoz de Cote, Differential evolution strategies for large-scale energy resource management in smart grids, in: Genetic and Evolutionary Computation Conference, 2017.

[50] C.A. Pea-Reyes, Evolutionary fuzzy modeling human diagnostic decisions, Ann. New York Acad. Sci. 1020 (2004) 190-211, http://dx.doi.org/10.1196/ annals.1310.017.

[51] S. Guillaume, Designing fuzzy inference systems from data: An interpretability-oriented review, IEEE Trans. Fuzzy Syst. 9 (3) (2001) 426-443.

[52] C. Ogwumike, M. Short, F. Abugchem, An embedded prototype of a residential smart appliance scheduling system, in: 2016 IEEE 21st International Conference on Emerging Technologies and Factory Automation, ETFA, 2016, pp. 1-5, http://dx.doi.org/10.1109/ETFA.2016.7733613.

[53] R. Khalid, N. Javaid, M.H. Rahim, S. Aslam, A. Sher, Fuzzy Energy management controller and scheduler for smart homes, Sustainable Computing: Informatics and Systems 21 (2019) 103-118, http://dx.doi.org/10. 1016/j.suscom.2018.11.010, http://www.sciencedirect.com/science/article/ pii/S2210537918302166.

[54] F. Wahid, D. Hyeun Kim, An efficient approach for energy consumption optimization and management in residential building using artificial bee colony and Fuzzy logic, Math. Probl. Eng. 2016 (2016) 1-13, http://dx.doi. org/10.1155/2016/9104735. 
[55] S. Hassan, A. Khosravi, J. Jaafar, M.A. Khanesar, A systematic design of interval type-2 fuzzy logic system using extreme learning machine for electricity load demand forecasting, Int. J. Electr. Power Energy Syst. 82 (2016) 1-10, http://dx.doi.org/10.1016/j.ijepes.2016.03.001, http://www. sciencedirect.com/science/article/pii/S0142061516303271.

[56] G.M. Biju, K.V. Shihabudheen, G.N. Pillai, Chaotic time series prediction using ELANFIS, in: 2017 6th International Conference on Computer Applications in Electrical Engineering-Recent Advances, CERA, 2017, pp. 473-478, http://dx.doi.org/10.1109/CERA.2017.8343376.

[57] L. Ciabattoni, F. Ferracuti, M. Grisostomi, G. Ippoliti, S. Longhi, Fuzzy Logic based economical analysis of photovoltaic energy management, Neurocomputing 170 (2015) 296-305.

[58] F. Chekired, A. Mahrane, Z. Samara, M. Chikh, A. Guenounou, A. Meflah, Fuzzy Logic energy management for a photovoltaic solar home, Energy Procedia 134 (2017) 723-730, http://dx.doi.org/10.1016/j.egypro.2017.09.566, http://www.sciencedirect.com/science/article/pii/S1876610217347008, Sustainability in Energy and Buildings 2017: Proceedings of the Ninth KES International Conference, Chania, Greece, 5-7 July 2017.

[59] D. Arcos-Aviles, F. Guinjoan, J. Pascual, L. Marroyo, P. Sanchis, R. Gordillo, P. Ayala, M.P. Marietta, A review of Fuzzy-based residential grid-connected microgrid energy management strategies for grid power profile smoothing, in: Energy Sustainability in Built and Urban Environments, Springer, 2019, pp. $165-199$.

[60] D. Arcos-Aviles, J. Pascual, L. Marroyo, P. Sanchis, F. Guinjoan, Fuzzy Logicbased energy management system design for residential grid-connected microgrids, IEEE Trans. Smart Grid PP (99) (2016) 1-1. http://dx.doi.org/ 10.1109/TSG.2016.2555245.

[61] M. Islam, F. Yang, C. Ekanayek, M. Amin, Grid power fluctuation reduction by fuzzy control based energy management system in residential microgrids, Int. Trans. Electr. Energy Syst. 29 (3) (2019) e2758, http://dx.doi.org/ 10.1002/etep.2758, arXiv:https://onlinelibrary.wiley.com/doi/pdf/10.1002/ etep.2758, https://onlinelibrary.wiley.com/doi/abs/10.1002/etep.2758.

[62] S. Leonori, E.D. Santis, A. Rizzi, F.M.F. Mascioli, Optimization of a microgrid energy management system based on a Fuzzy logic controller, in: IECON 2016 - 42nd Annual Conference of the IEEE Industrial Electronics Society, 2016, pp. 6615-6620, http://dx.doi.org/10.1109/IECON.2016.7793965.

[63] J.P. Fossati, A. Galarza, A. Martín-Villate, J.M. Echeverría, L. Fontán, Optimal scheduling of a microgrid with a fuzzy logic controlled storage system, Int. J. Electr. Power Energy Syst. 68 (2015) 61-70, http://dx.doi.org/10.1016/j.ijepes.2014.12.032, http://www.sciencedirect. com/science/article/pii/S0142061514007595.
[64] S. Leonori, A. Martino, A. Rizzi, F.M.F. Mascioli, ANFIS Synthesis by clustering for microgrids EMS design, in: Proceedings of the 9th International Joint Conference on Computational Intelligence - Volume 1: IJCCI, SciTePress, 2017, pp. 328-337, http://dx.doi.org/10.5220/ 0006514903280337.

[65] S. Leonori, E.D. Santis, A. Rizzi, F.M.F. Mascioli, Multi objective optimization of a fuzzy logic controller for energy management in microgrids, in: 2016 IEEE Congress on Evolutionary Computation (CEC), 2016, pp. 319-326, http://dx.doi.org/10.1109/CEC.2016.7743811.

[66] E. De Santis, A. Rizzi, A. Sadeghian, Hierarchical genetic optimization of a fuzzy logic system for energy flows management in microgrids, Appl. Soft Comput. J. 60 (2017) 135-149, http://dx.doi.org/10.1016/j.asoc.2017. 05.059 .

[67] O. Cordón, F. Herrera, F. Hoffmann, L. Magdalena, Genetic Fuzzy systems: Evolutionary tuning and learning of Fuzzy knowledge bases, first ed., in: Advances in Fuzzy Systems-Applications and Theory, vol. 19, World Scientific Publishing Co. Pte. Ltd., 2001.

[68] H. Jaeger, The "echo state" approach to analysing and training recurrent neural networks-with an erratum note, Bonn, Germany: German National Research Center for Information Technology GMD Technical Report, 148 (34) 2001, p. 13.

[69] M. Lukoševičius, H. Jaeger, Reservoir computing approaches to recurrent neural network training, Comp. Sci. Rev. 3 (3) (2009) 127-149, http://dx.doi.org/10.1016/j.cosrev.2009.03.005, http://www.sciencedirect. com/science/article/pii/S1574013709000173.

[70] M. Lukoševičius, A practical guide to applying echo state networks, in: G. Montavon, G.B. Orr, K.-R. Müller (Eds.), Neural Networks: Tricks of the Trade, second ed., Springer Berlin Heidelberg, Berlin, Heidelberg, 2012, pp. 659-686.

[71] M. Grant, S. Boyd, CVX: Matlab software for disciplined convex programming, version 2.1, 2014, http://cvxr.com/cvx.

[72] M. Grant, S. Boyd, Graph implementations for nonsmooth convex programs, in: V. Blondel, S. Boyd, H. Kimura (Eds.), Recent Advances in Learning and Control, in: Lecture Notes in Control and Information Sciences, Springer-Verlag Limited, 2008, pp. 95-110, http://stanford.edu/ boyd/graph_dcp.html. 